\title{
Use of prophylactic uterotonics during the third stage of labor: a survey of provider practices in community health facilities in Sierra Leone
}

Abirami Natarajan ${ }^{1,2^{*}}$, Roy Ahn ${ }^{1,2}$, Brett D. Nelson ${ }^{1,2}$, Melody Eckardt $^{1}$, Jennifer Kamara ${ }^{1}$, SAS Kargbo $^{3}$, Pity Kanu ${ }^{3}$ and Thomas F. Burke ${ }^{1,2}$

\begin{abstract}
Background: Postpartum hemorrhage remains the leading cause of maternal mortality worldwide. Administration of uterotonics during the third stage of labor is a simple and well established intervention that can significantly decrease the development of postpartum hemorrhage. Little is known about the use of prophylactic uterotonics in peripheral health centers, where the majority of normal deliveries occur. The purpose of this study is to assess health provider current practices and determinants to the use of prophylactic uterotonics in Sierra Leone, a country with one of the highest maternal mortality ratios worldwide.
\end{abstract}

Methods: This is a mixed methods study using descriptive cross-sectional survey and qualitative interviews in community health facilities in Freetown, Sierra Leone following a comprehensive training on postpartum hemorrhage. Facilities and providers were surveyed between May and June 2014. Qualitative methods were used to identify barriers and facilitators to the use of prophylactic uterotonics.

Results: A total of 134 providers were surveyed at 39 periphreal health facilities. Thirteen facilities (39\%) reported an inconsistent supply of oxytocin. The majority of facilities (64\%) stored oxytocin at room temperature. Provider level, in-service training, and leadership role were significantly associated with prophylactic uterotonic use. Overall, $62 \%$ of providers reported routine use. Midwives were most likely to routinely administer uterotonics (93\%), followed by community health officers/assistants (78 \%), maternal and child health aides (56\%), and state-enrolled community health nurses (52\%). Of the providers who received in-service training, $67 \%$ reported routine use; of those with no in-service training, $42 \%$ reported routine use. Qualitative analysis revealed that facility protocols, widespread availability, and provider perception of utility facilitated routine use. Common barriers reported included inconsistent supply of uterotonics, lack of knowledge regarding timely administration, and provider attitude regarding utility of uterotonics following normal deliveries.

Conclusion: There is considerable room for improvement in availability and administration of prophylactic uterotonics. Understanding barriers to routine use may aid in developing multifaceted pre-service and in-service training interventions designed to improve routine intrapartum care.

Keywords: Prophylactic uterotonics, Postpartum hemorrhage, Sierra Leone

\footnotetext{
* Correspondence: abirami_natarajan@hms.harvard.edu

'Division of Global Health and Human Rights, Department of Emergency

Medicine, Massachusetts General Hospital, Zero Emerson Place Suite 104,

Boston, MA 02114, USA

${ }^{2}$ Harvard Medical School, 25 Shattuck Street, Boston, MA 02115, USA

Full list of author information is available at the end of the article
} 


\section{Background}

Postpartum hemorrhage $(\mathrm{PPH})$ is the most common cause of maternal mortality and morbidity worldwide, with the majority of $\mathrm{PPH}$-related deaths occurring in low-income countries [1-3]. Active management of the third stage of labor (AMTSL) is a well-established protocol that has been shown to significantly reduce the incidence of PPH $[4,5]$. AMTSL consists of administration of a prophylactic uterotonic after delivery of the newborn, fundal massage, delayed cord clamping and controlled cord traction. The initial studies evaluating the efficacy of AMTSL studied these components as a package. Although there is strong evidence to recommend the use of prophylactic uterotonic for the prevention of $\mathrm{PPH}$, the relative importance of controlled cord traction and fundal massage is uncertain $[6,7]$. Based on this, the International Federation of Gynecologists and Obstetricians (FIGO) and the World Health Organization (WHO) recommend routine use of uterotonics to prevent $\mathrm{PPH}[8,9]$.

Despite strong evidence supporting the effectiveness of these guidelines, there remains significant inter-country variation in rates of AMTSL uptake. A WHO crosssectional survey of 15 university-based obstetric centers in both developing and developed countries found low observed use of AMTSL, occurring on average in only $25 \%$ of deliveries [10]. Other studies investigating both AMTSL practices and interventions targeted at improving AMTSL adherence in the hospital setting have also found low rates of knowledge and application [11-14].

Most research has understandably focused on highvolume facilities or complicated deliveries; however, little is known about AMTSL practices in primary health facilities in particularly low income countries, where normal uncomplicated deliveries are encouraged [15]. These facilities are fundamentally different from hospitals in that they employ lower-skilled providers, have limited access to health information, and have fewer resources to manage the consequence of $\mathrm{PPH}$. However, given the role of preventive practices, understanding provider adherence to interventions that prevent $\mathrm{PPH}$ at these facilities can provide insight into opportunities for improving routine intrapartum care - a cornerstone strategy for addressing maternal mortality [16].

The purpose of this study is to gain further insight into provider use of prophylactic uterotonics following a comprehensive PPH-training package in Freetown, Sierra Leone. We sought to understand the personal practice of prophylactic uterotonic administration in providers conducting routine deliveries. Furthermore, we sought to understand if factors such as in- service AMTSL training, provider cadre, and leadership positions were associated with the routine use of uterotonics. In addition, qualitative methods were used to identify facilitators and barriers to routine use of prophylactic uterotonics.

\section{Methods}

In December 2013, Massachusetts General Hospital in conjunction with the Sierra Leone Ministry of Health conducted a total of eight three-hour workshops on a PPH in-service training called Every Second Matters for Mothers and Babies - Uterine Balloon Tamponade (ESM-UBT). The training components of ESM-UBT included AMTSL (administration of 10 IU oxytocin IM or $\mathrm{IV}$, fundal massage, delayed cord clamping and controlled cord traction), basic PPH management, and the use of condom-catheter uterine balloon tamponade (UBT) as a second-line treatment for uncontrolled PPH [17]. Two representatives from each of 46 peripheral health facilities - usually a facility head and an experienced midwife - were asked to attend this session and subsequently disseminate the knowledge to all members of the facility who are involved in conducting deliveries. Following the central training, these representatives referred to as "UBT champions" - were contacted via phone to ensure that all members of the facility were trained. Each facility was provided with two PPH instruction manuals, a pictorial wall chart, and condom-catheter UBT kits. By March 2014, all of the original facility representatives reported that all members of their facility had been trained.

This was a descriptive cross-sectional survey of reported uterotonic use during the third stage of labor for providers working in peripheral health care facilities. This survey was embedded within a larger qualitative study assessing facilitators and barriers to uptake of the ESM-UBT training package (results reported separately). During May - June 2014, approximately 6 months after the central training session, a survey was conducted at 39 of the $42(92.9 \%)$ peripheral health facilities that attended the initial central training. Three facilities were not visited because of difficult terrain.

At each facility, information regarding the availability and storage of uterotonics was obtained from facility or maternity heads. Since oxytocin is the primary uterotonic used and freely distributed by the Sierra Leone government, providers were specifically asked if they had a consistent supply of oxytocin available at their facility. The heads of the facility were then asked to identify all members of the facility who conduct deliveries on that particular shift. All providers who conduct deliveries in primary health facilities in Sierra Leone are authorized to use oxytocin during the third stage of labor per the Sierra Leone national protocol for the prevention and treatment of $\mathrm{PPH}$. Each provider was asked general questions about their professional training, in-service training in $\mathrm{PPH}$ (including AMTSL), years of experience, 
and delivery volume. Providers were then asked about their personal practice of managing the third stage of labor during vaginal deliveries. Qualitative interviews were conducted to capture provider practices, attitudes, and perceived barriers to routine use of prophylactic uterotonics. Verbal informed consent was obtained from all participants. All data were recorded and entered into a Microsoft Excel spreadsheet. Clinical results are reported as mean \pm SEM. Analyses between groups for statistically significant differences in categorical data were performed with the Fisher's Exact and Fisher-FreemanHalton Tests. Student's t-tests were used to assess differences among groups with continuous variables ( $R$ version 3.1.1).

Ethical approval for this study was obtained from the Partners Healthcare Human Research Committee (12/ 18/2013 protocol\#: 2012P002112/MGH) and Sierra Leone Ministry of Health and Sanitation (05/12/2014).

\section{Results}

A total of 134 providers were surveyed at 39 study sites (1-8 interviews per site). Table 1 shows the characteristics of health facilities, including average delivery volume, oxytocin availability, and oxytocin storage. The majority of facilities (62\%) were CHCs, followed by CHPs (26 \%), and MCHPs (13\%). Oxytocin was most consistently available at MCHPs (80\%), followed by CHP (60 \%), and CHCs (58 \%). Fifteen facilities (44\%) reported shortages of oxytocin. The majority of facilities (64 \%) reported storing oxytocin at room temperature.

Table 2 shows the characteristics of providers interviewed. Providers reported conducting between 1 and 100 deliveries per month with a mean (SD) of 20 (19.2) deliveries per month. Their experience ranged from 6 months to 32 years, with a mean (SD) of 7.2 (6.7) years. The majority of respondents was female (90\%). MCHAs (51 \%) and SECHNs (25\%) accounted for the majority of respondents. Thirteen providers $(21 \%)$ held administrative positions - either head of facility or maternity ward. The majority of providers $(77 \%)$ received targeted training in AMTSL since their professional training.

When providers were asked about their management of the third stage of labor, 83 (62\%) of the 134 providers

Table 1 Characteristics of health facilities

\begin{tabular}{llll}
\hline $\begin{array}{l}\text { Facility } \\
\text { type } \\
(n=39)\end{array}$ & $\begin{array}{l}\text { Average number } \\
\text { of deliveries in } \\
\text { facility/month }\end{array}$ & $\begin{array}{l}\text { Oxytocin } \\
\text { availability } \\
\text { (\% always available) }\end{array}$ & $\begin{array}{l}\text { Oxytocin } \\
\text { storage } \\
(\% \text { stored in fridge) }\end{array}$ \\
\hline $\operatorname{MCHP}(n=5)$ & 21 & $4(80 \%)$ & $0(0.0 \%)$ \\
CHP $(n=10)$ & 31 & $6(60 \%)$ & $2(20 \%)$ \\
CHC $(n=24)$ & 50 & $14(58 \%)$ & $11(46 \%)$ \\
\hline
\end{tabular}

CHP community health post, MCHP maternal and child health post, $C H C$ community health center
Table 2 Characteristics of obstetric providers interviewed

\begin{tabular}{|c|c|c|}
\hline Characteristics & Number & Percent (\%) \\
\hline \multicolumn{3}{|l|}{ Experience (years) } \\
\hline $0-9$ & 97 & 72 \\
\hline $10-19$ & 27 & 20 \\
\hline $20-29$ & 6 & 5 \\
\hline$>30$ & 4 & 3 \\
\hline \multicolumn{3}{|l|}{ Gender } \\
\hline Male & 13 & 10 \\
\hline Female & 121 & 90 \\
\hline \multicolumn{3}{|l|}{ Cadre } \\
\hline Midwife & 14 & 11 \\
\hline SECHN & 34 & 25 \\
\hline $\mathrm{MCHA}$ & 68 & 51 \\
\hline $\mathrm{CHO} / \mathrm{CHA}$ & 18 & 13 \\
\hline \multicolumn{3}{|l|}{ Leadership* } \\
\hline Yes & 28 & \\
\hline No & 106 & 21 \\
\hline AMTSL training & & 79 \\
\hline In-service AMTSL training & 103 & 77 \\
\hline No in-service training & 31 & 23 \\
\hline \multicolumn{3}{|c|}{ Provider average deliveries/month } \\
\hline $1-19$ & 81 & 60 \\
\hline $20-39$ & 32 & 24 \\
\hline $40-59$ & 13 & 10 \\
\hline $60-79$ & 5 & 4 \\
\hline 80-100 & 3 & 2 \\
\hline
\end{tabular}

*Leadership position includes providers in charge of the facility or in charge of the labor and delivery room

reported that they routinely give prophylactic uterotonics. With the exception of one provider who used misoprostol, all providers who reported uterotonic use reported using oxytocin. The relationship between provider characteristics and reported use of uterotonics is outlined in Table 3. Provider level, in-service training, leadership roles, and years of experience were significantly associated with reported use of uterotonics $(\mathrm{p}<0.05)$. Midwives were most likely to administer oxytocin (93\%), followed by $\mathrm{CHO} /$ CHA (78 \%), MCHAs (56 \%), and SECHNs (53\%). Providers who received a variation of in-service training were more likely to report administration of uterotonics that those who did not $(\mathrm{p}<0.01)$. Of the 103 providers who had in-service training, 69 (67\%) gave uterotonics. Less than half ( $42 \%)$ of the 31 providers who did not receive in-service training administered uterotonics during the third stage of labor. Providers who administered uterotonics on average had 8.5 years of experience (SEM 0.87) compared with those who did not report using uterotonics, who had 5.1 (SEM 0.43) years of experience. 
Table 3 Relationship between provider characteristics and reported routine administration of prophylactic uterotonics

\begin{tabular}{lll}
\hline $\begin{array}{l}\text { Provider level } \\
\text { of training }\end{array}$ & $\begin{array}{l}\text { Number (percentage) who } \\
\text { report routinely administered } \\
\text { prophylactic uterotonic }\end{array}$ & $P<0.05$ \\
MCHA & $38 / 68(56 \%)$ & \\
SECHN & $18 / 34(53 \%)$ & \\
Midwife & $13 / 14(93 \%)$ & \\
CHO/CHA & $14 / 18(78 \%)$ & $P<0.01$ \\
Total & $83 / 134(62 \%)$ & \\
Type of training & & \\
In service training & $69 / 103(67 \%)$ & \\
No in-service training & $13 / 31(42 \%)$ & \\
\hline
\end{tabular}

Table 4 Representative quotes regarding facilitators and barriers to the use of prophylactic uterotonics during the third stage of labor Facilitators

Providers who understood the value of prophylactic uterotonics as a life saving drug purchased oxytocin when the government supply was inconsistent.

- "Because oxy is an emergency drug it is very important for delivery. Sometime it is short, but I buy and keep it in the cupboard. Yea but sometime government have shortage and don't give us, but we buy." - Clinical Health Officer

- "I want to save life, I'm not going to depend on government to give me oxy, so that's why we usually buy it, whenever we have a case. "- Midwife

Established protocols in facility for preventing PPH

- "One of the protocols is that when the woman has delivered we give oxytocin.... All of this management is an attempt to prevent PPH." - Clinical Health Officer

Providers who understand the importance of oxytocin as a life saving drug strongly encouraged patients to purchase oxytocin when it was unavailable

- "When we are short of oxytocin, we buy. If there is nothing, we tell them [patients], when they are term, birth preparedness, this will one of the things that will be in the kit. You bring this, you bring this, and you bring this. The nurses write for them to bring. But normally it is around. For those who can't afford, we give. "- Clinical Health Officer

. "We ask them [patients], we force them to buy it because you know after delivery we need to help them with oxytocin." - midwife

Providers purchase oxytocin out of fear of being audited

- "They order it so that we can save our own selves, because if you have a maternal death and you are on duty, they will query you. They will judge you and you have to prove yourself, you have to take all of your documents and explain what happened and what did not happen. So you will not allow that. So even if the midwife is not around, we have to take it out of [our own money]." - Maternal and Child Health Aid

Barriers

Providers reported giving uterotonics when it was available, but during shortages managed the third stage expectantly.

- "We give oxytocin if we have, but if we don't have then we express normal procedures [expectant management]." - MCHA

Misconceptions about the universal indication of uterotonics - such as prolonged labor or if there is difficulty delivering placenta - prevented providers from giving routinely

- "At times - we do not give oxytocin if the placenta comes out easily. There is no need to give oxytocin."

- "Only when it is difficult labor we are told to give. But we haven't had any yet.

Some providers report learning that uterotonics should be given to all patients but believe that it is not required unless the mother is bleeding

- "Well because we are supposed to give, but when we don't have the case and we are not seeing enough profuse bleeding, I do not see justification to give. So normally we do fundal massage for contractions to take place, and if there are clots then we expel. We check for tear, perineal, cervical and we see how best - based on that if there is no profuse bleeding we don't give." - Clinical Health officer

Belief that prophylactic oxytocin is not needed if a patient delivers normally

- "After the delivery I didn't give oxytocin because she delivered normally. Only if she is bleeding. Because some of them they deliver normally, no problem." - MCHA

- "After delivery I do not give any oxytocin because she delivered normally. Later on after 30-40 min \{inaudible\} so I had to set my normal saline with $20 \mathrm{IU}$ oxytocin and the woman was still bleeding so I called for sister to help me." - MCHA 
patient. A very small number of providers also expressed fear of being audited by the government in the case of a poor maternal outcome as a driver to purchasing backup supplies.

Barriers to the consistent use of prophylactic uterotonics included inconsistent supply and correct knowledge regarding proper indications for administration. Providers expressed significant concern when oxytocin was unavailable at their facility. Some providers reported the use of uterotonics during the third stage of labor when it was available, but resorted to expectant management when the government supply of uterotonics was unavailable and patients could not purchase it due to either cost or access. Gaps in knowledge regarding the proper indication and utility of prophylactic uterotonics prevented providers from its routine administration. Several providers expressed their belief that women who underwent normal labor did not require prophylactic uterotonics following delivery of the newborn. Other providers specified that they would provide prophylactic uterotonics if a patient's labor was prolonged, there were challenges delivering the placenta, or if there was any bleeding during or immediately after delivery. Several providers reported that they were taught both in their traditional and in-service training to give prophylactic uterotonics routinely to all women during the third stage of labor, but they did not see a justification for this if a woman was not bleeding, and therefore did not routinely administer it.

\section{Discussion}

Our results showed that although there is a widespread awareness, acceptance, and use of prophylactic uterotonics in Sierra Leone, there remains considerable room for improvement. Of the 134 providers surveyed, $62 \%$ reported routine use of uterotonics during the third stage of labor. Although all health cadres are taught AMTSL during pre-service training, those who received in-service training including AMTSL were significantly more likely to administer prophylactic uterotonics than those who did not.

Providers in leadership positions such as $\mathrm{CHOs}$ and midwives were also more likely to report uterotonic use than providers who are frontline in conducting normal uncomplicated deliveries (SECHNs and MCHAs). Ninetythree percent of midwives and $77 \%$ of $\mathrm{CHOs} / \mathrm{CHAs}$ reported routine use of uterotonics. These findings may reflect differences in initial training or provider level of experience. Unfortunately, providers with more experience such as midwives and $\mathrm{CHOs} / \mathrm{CHAs}$ are more likely to be involved after PPH has already developed, rather than during the routine management of labor. This finding, however, does represent a strong basis for transfer of knowledge within a facility. Quality improvement interventions focused on the active guideline development by facility leaders - which has shown promise in other settings - may play a role in increasing routine uterotonic use [18].

Consistent provision of drugs is required for the timely administration of any intervention. Although oxytocin is an essential drug freely supplied by the Sierra Leone government, providers cite that inadequate supply of uterotonics remains a significant challenge to routine administration. Our results showed that $39 \%$ of facilities had an inconsistent supply of oxytocin. Furthermore, only one-third of facilities stored oxytocin within the manufacturer-recommended temperature of $2-8^{\circ}$. Although providers who perceived the value of oxytocin purchased it with their own funds or strongly encouraged patients to do so, many providers reported that oxytocin shortages contributed to their use of expectant management of the third stage of labor. Limited availability of oxytocin in primary health facilities likely also has implications for the emergent treatment of postpartum hemorrhage. Further research is needed to assess the efficacy of oxytocin in room temperature conditions as well as the role of temperature independent uterotonics such as misoprostol in resource limited settings.

\section{Study strengths and limitations}

The major strength of this study is the location. Given that Sierra Leone carries one of the highest maternal mortality ratios worldwide, understanding provider practices of preventive interventions in peripheral health facilities where routine deliveries are encouraged serves as a basis for targeted quality improvement. In addition, few studies have documented specific provider perceptions that remain barriers to routine prophylactic uterotonic use. Limitations of this study include its descriptive nature and small sample size. Interviews are subject to recall, and given desirability bias, providers may over-report their use of oxytocin. Furthermore, this study did not assess the timing of oxytocin administration. Given that uterotonics are recommended within one minute after delivery of the newborn, it is likely that observed deliveries in these facilities would be less than optimal. Observed studies conducted in high-volume hospitals have indeed found a discrepancy between provider knowledge and timely administration of prophylactic uterotonics [19]. However, observing deliveries in peripheral facilities with lower deliveries presents logistical and financial challenges.

\section{Conclusion}

Despite evidence-based guidelines recommending the use of prophylactic uterotonics during the third stage of labor, hospitals in both developed and developing countries significantly underuse it. This study documents 
provider practices administering prophylactic uterotonics in lower-level facilities in Sierra Leone, and provides insight into several underlying reasons for its underutilization. Although $67 \%$ of providers who underwent in-service training reported the use of uterotonics, there still remains a gap in optimal translation from in-service training to knowledge retention and clinical practice. Other studies have documented this, as well as the need for multiple components in addition to dissemination of knowledge for clinical behavior change to occur $[11,12]$. Further research is needed to determine the optimal duration, components, and delivery of targeted AMTSL training.

Among the providers who did not routinely administer uterotonics, supply of drugs, provider knowledge of proper indication, and provider attitudes toward the utility of prophylactic uterotonics were the most common barriers to routine administration. For instance, several providers described their belief that mothers who underwent normal deliveries or did not have bleeding did not require prophylactic uterotonics. This belief may lead to sporadic use of prophylactic uterotonics, particularly in the context of drug shortages. Professional and inservice training presents an opportunity to emphasize and reinforce the value of routine administration of uterotonics even in normal deliveries.

There remains considerable room for quality improvement in administering prophylactic uterotonics during the third stage of labor. Although inconsistent supply of uterotonics remains a barrier to routine use, accurate provider knowledge regarding indications for and value of prophylactic uterotonics also contribute to its underutilization. Emphasis on incorporating and emphasizing the use of uterotonics during pre-service and inservice training and the development of facility protocols may improve practices. Consistent availability and implementation of oxytocin during the third stage of labor represents a very simple, feasible, inexpensive intervention to decrease bleeding postpartum, the most common cause of maternal mortality worldwide.

\section{Abbreviations \\ AMTSL: Active management of the third stage of labor; PPH: Post partum hemorrhage; UBT: Uterine balloon tamponade; SECHN: State enrolled child health nurses; MCHA: Maternal and child health aides; $\mathrm{CHO}$ : Clinical health officers; FIGO: International Federation of Gynecologists and Obstetricians; ICM: International Confederation of Midwives; WHO: World Health Organization.}

\section{Competing interests}

All authors have no conflict of interest.

\section{Authors' contributions}

$T B, M E, B N, R A$, and $A N$ contributed to the conception of the study and participated in study design. TB, ME, PK, JK, RA, and SK participated in implementation of study. TB, ME, PK, and JK participated in field trainings. AN and JK participated in collection of field data. AN, BN, ME, RA, and TB participated in data analysis and interpretation of results. AN drafted the manuscript with significant input, review, and editing from all authors. All authors accept overall content for the report of the work.

\section{Acknowledgments}

We would like to thank all of the health care providers who volunteered their time to work as UBT champions and all health care providers who participated in this study. We thank Tom Chittenden for his help with the statistical analysis. Funding was provided by Saving Lives at Birth

(Washington, DC, USA), Humanitarian Innovation Fund, Ujenzi Charitable Trust, and Harvard Medical School (Boston, MA, USA).

\section{Author details}

'Division of Global Health and Human Rights, Department of Emergency Medicine, Massachusetts General Hospital, Zero Emerson Place Suite 104, Boston, MA 02114, USA. ${ }^{2}$ Harvard Medical School, 25 Shattuck Street, Boston, MA 02115, USA. ${ }^{3}$ Ministry of Health and Sanitation, 4th Floor Youyi Building, Freetown, Sierra Leone.

Received: 4 April 2015 Accepted: 21 January 2016 RH?

\section{References}

1. Khan KS, Wojdyla D, Say L, Gulmezoglu AM, Van Look PF. WHO analysis of causes of maternal death: a systematic review. Lancet. 2006:367(9516):1066-74.

2. AbouZahr C. Global burden of maternal death and disability. Br Med Bull. 2003:67:1-11.

3. Kassebaum NJ, Bertozzi-Villa A, Coggeshall MS, Shackelford KA, Steiner C, Heuton KR, et al. Global, regional, and national levels and causes of maternal mortality during 1990-2013: a systematic analysis for the Global Burden of Disease Study 2013. Lancet. 2014:384(9947):980-1004.

4. Begley CM, Gyte GM, Devane D, McGuire W, Weeks A. Active versus expectant management for women in the third stage of labour. Cochrane Database Syst Rev. 2011;11:CD007412

5. Rogers J, Wood J, McCandlish R, Ayers S, Truesdale A, Elbourne D. Active versus expectant management of third stage of labour: the Hinchingbrooke randomised controlled trial. Lancet. 1998;351(9104):693-9.

6. Westhoff G, Cotter AM, Tolosa JE. Prophylactic oxytocin for the third stage of labour to prevent postpartum haemorrhage. Cochrane Database Syst Rev. 2013;10:CD001808.

7. Gulmezoglu AM, Lumbiganon P, Landoulsi S, Widmer M, Abdel-Aleem H, Festin $\mathrm{M}$, et al. Active management of the third stage of labour with and without controlled cord traction: a randomised, controlled, non-inferiority trial. Lancet. 2012;379(9827):1721-7.

8. International Confederation of $M$, International Federation of $G$, Obstetricians. Joint statement: management of the third stage of labour to prevent post-partum haemorrhage. J Midwifery Womens Health. 2004:49(1):76-7.

9. WHO recommendations for the prevention and treatment of postpartum hemorrhage, 2012. Available at: http://apps.who.int/iris/bitstream/10665/ 75411/1/9789241548502_eng.pdf.

10. Festin MR, Lumbiganon P, Tolosa JE, Finney KA, Ba-Thike K, Chipato T, et al. International survey on variations in practice of the management of the third stage of labour. Bull World Health Organ. 2003;81(4):286-91.

11. Oladapo OT, Akinola Ol, Fawole AO, Adeyemi AS, Adegbola O, Loto OM, et al. Active management of third stage of labor: evidence versus practice. Acta Obstet Gynecol Scand. 2009:88(11):1252-60.

12. Cherine M, Khalil K, Hassanein N, Sholkamy H, Breebaart M, Elnoury A. Management of the third stage of labor in an Egyptian teaching hospital. Int J Gynaecol Obstet. 2004;87(1):54-8.

13. Fiigueras A, Narvaez E, Valsecia M, Vasquez S, Rojas G, Camilo A, et al. An education and motivation intervention to change clinical management of the third stage of labor - the GIRMMAHP Initiative. Birth. 2008:35(4):283-90.

14. Althabe F, Buekens P, Bergel E, Belizan JM, Campbell MK, Moss N, et al. A behavioral intervention to improve obstetrical care. N Engl J Med. 2008;358(18):1929-40.

15. Smit M, Van Stralen G, Wolterbeek R, Van Dilen J, Van Roosmajen J, Slootweg Y. Survey of prophylactic use of uterotonics in the third stage of labour in the Netherlands. Midwifery. 2013:29(8):859-62.

16. Campbell OM, Graham WJ. Lancet Maternal Survival Series steering g. Strategies for reducing maternal mortality: getting on with what works. Lancet. 2006;368(9543):1284-99. 
17. Nelson BD, Stoklosa H, Ahn R, Eckardt MJ, Walton EK, Burke TF. Use of uterine balloon tamponade for control of postpartum hemorrhage by community-based health providers in South Sudan. Int J Gynaecol Obstet. 2013;122(1):27-32

18. Chaillet N, Dube E, Dugas M, Audibert F, Tourigny C, Fraser WD, et al. Evidence-based strategies for implementing guidelines in obstetrics: a systematic review. Obstet Gynecol. 2006;108(5):1234-45.

19. Stanton CK, Deepak NN, Mallapur AA, Katageri GM, Mullany LC, Koski A, et al Direct observation of uterotonic drug use at public health facilitybased deliveries in four districts in India. Int J Gynaecol Obstet. 2014; 127(1):25-30.

Submit your next manuscript to BioMed Central and we will help you at every step:

- We accept pre-submission inquiries

- Our selector tool helps you to find the most relevant journal

- We provide round the clock customer support

- Convenient online submission

- Thorough peer review

- Inclusion in PubMed and all major indexing services

- Maximum visibility for your research

Submit your manuscript at www.biomedcentral.com/submit 